\title{
Correspondence:
}

\section{Transmission risk of patients with COVID-19 meeting discharge criteria should be interpreted with caution}

Jun-wei SU, Wen-rui WU, Guan-jing LANG, Hong ZHAO, Ji-fang SHENG ${ }^{\dagger+}$

Department of Infectious Diseases, State Key Laboratory for the Diagnosis and Treatment of Infectious Diseases, Collaborative Innovation Center for the Diagnosis and Treatment of Infectious Diseases, National Clinical Research Center for Infectious Diseases, the First Affiliated Hospital, School of Medicine, Zhejiang University, Hangzhou 310003 , China

†E-mail: jifang_sheng@zju.edu.cn

https://doi.org/10.1631/jzus.B2000117

Published online May 8, 2020

As of Apr. 22, 2020, the World Health Organization (2020) has reported over 2.4 million confirmed coronavirus disease 2019 (COVID-19) patients and 169151 deaths. Recent articles have uncovered genomic characteristics and clinical features of COVID-19 (Chan et al., 2020; Chang et al., 2020; Guan et al., 2020; Zhu et al., 2020), while our understanding of COVID-19 is still limited. As suggested by guidelines promoted by the General Office of National Health Commission of the People's Republic of China (2020) (from Versions 1 to 6), discharged standards for COVID-19 were still dependent on viral real-time polymerase chain reaction (RT-PCR) tests of respiratory specimens, showing that recovered COVID-19 patients with twice negative RT-PCR could meet discharge criteria. Here, we examined two cases in which nucleic acid test results were inconsistent with clinical and radiological findings, leading to suboptimal care.

\footnotetext{
¿ Corresponding author

* Project supported by the National Natural Science Foundation of China (No. 81670567) and the National Science and Technology Major Project of China (No. 2018ZX10715014-004-002)

(b) ORCID: Jun-wei SU, https://orcid.org/0000-0002-1762-1079; Ji-fang SHENG, https://orcid.org/0000-0003-0732-1555

(c) Zhejiang University and Springer-Verlag GmbH Germany, part of Springer Nature 2020
}

We enrolled two cases of COVID-19 who met the discharge criteria posted by the guideline as follows: (1) afebrile maintained for at least $3 \mathrm{~d}$; (2) respiratory symptoms improved significantly; (3) viral nucleic acid test being negative at least twice (each interval greater than $24 \mathrm{~h}$ ); (4) lung image showing that lesions were significantly improved or completely absorbed; and (5) no oxygen therapy and pulse oxygen saturation $\left(\mathrm{SpO}_{2}\right)$ of $>93 \%$. In concern of transmission risk of discharged patients, another detection of severe acute respiratory syndrome coronavirus 2 (SARS-CoV-2) was done before discharge, while both of the RT-PCR results were positive. Patients' data were collected from the First Affiliated Hospital, School of Medicine, Zhejiang University, Hangzhou, China. Chest computed tomography (CT) was performed. Sputum was sampled for detection of SARSCoV-2 through RT-PCR (Shanghai BioGerm Medical Biotechnology Co., Ltd., China). Approval was obtained from the ethics committee and informed consent was signed by the patients.

Patient 1, a 40-year-old female, presented with fever and a mild dry cough on Jan. 17, 2020 (Day -4) after contact with a colleague from Wuhan, China, $4 \mathrm{~d}$ previously, who was confirmed to have COVID-19. She was diagnosed with COVID-19 in our center on Jan. 21 (Day 1) with a positive RT-PCR test in sputum and bilateral infiltration lesions on chest $\mathrm{CT}$ images (Fig. 1a). She was treated with lopinavir/ritonavir (LPV/r) and Arbidol combined with methylprednisolone and was afebrile by Day 6. Sputum was sampled daily for SARS-CoV-2 detection and the results turned negative on Day 8 and Day 10. Lung CT scan on Day 9 showed reduced infusion lesions (Fig. 1b). However, a weak positive result of SARS-CoV-2 in sputum was demonstrated on Day 11, and remained positive again from Day 12 to Day 14. This patient was discharged on Day 17. We followed up this patient in 
clinic at time points of one week, two weeks, and four weeks after discharge. RT-PCR results of sputum and stool were negative.

Patient 2, a 50-year-old male, had fever for $8 \mathrm{~d}$ and was confirmed to have COVID-19 on Jan. 23, 2020 (Day 1) in our center. He was a resident in Wuhan and travelled to Hangzhou. His chest CT on Jan. 24 (Day 2) showed bilateral ground-glass and consolidative lesions (Fig. 1c). After treatment with LPV/r and Arbidol combined with methylprednisolone, his temperature returned to normal on Day 5 and detection of SARS-CoV-2 turned negative from Day 8 to Day 9 with synchronized improvement in chest CT on Day 10 (Fig. 1d). Unexpectedly, RT-PCR results were positive on Day 10 to Day 12 in this patient. RT-PCR results turned negative from Day 13 to Day 16 while a positive result was detected again on Day 17. This patient was discharged on Day 21. We followed up this patient in clinic at time points of one week, two weeks, and four weeks after discharge. RT-PCR results of sputum and stool were negative.
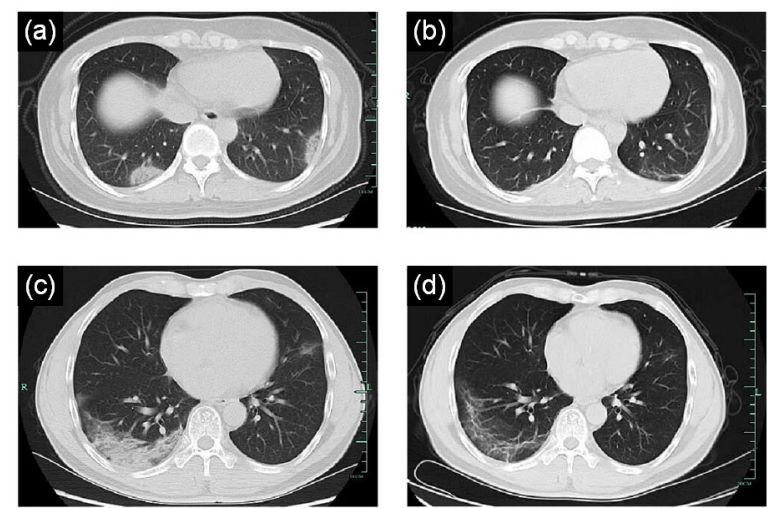

Fig. 1 Chest CT images of two patients with COVID-19 (a) Chest computed tomography (CT) image of Patient 1 on admission presents multiple ground-glass opacities distributed in the periphery of inferior lobe of both lungs. (b) Rechecked chest CT image of Patient 1 on Day 9 shows residual bronchovascular shadows. (c) Chest CT of Patient 2 on Day 2 shows diffuse confluent and patchy ground-glass and consolidative opacity in the right lower lung and ground-glass opacity in the inferior lingual of left upper lobe. (d) Rechecked chest $\mathrm{CT}$ of Patient 2 on Day 10 shows remarkable absorption of lesions

Thus, in Patients 1 and 2, although lung images had apparently improved with continuously negative RT-PCR results and patients met the criteria of discharge, positive results repeatedly emerged in sub- sequent respiratory specimens. Prolonged excretion of virus was also reported in SARS-CoV (Fang et al., 2004). Our study describes two cases with subsequently positive nucleic acid results in patients of COVID-19 who met discharge criteria and this raises concern about the transmission risk associated with discharged COVID-19 patients.

One potential explanation for the discrepant results from the RT-PCR of respiratory specimens is that the burden of SARS-CoV-2 is much higher in the lower respiratory tract rather than in the upper (Huang et al., 2020). Therefore, inappropriate sampling of throat swabs or oral fluid instead of deep respiratory specimens may lead to inadequate sampling and low or variable detection rates. Also, patients who present with dry cough or are in the recovery stage rarely have expectorated sputum. Deep lung tissue specimens from induced sputum or bronchoalveolar lavage fluid are also impractical given the limited resources and risk of transmission. Thus, it is important to establish repeated sampling following standardized procedures and optimized detection methods at each treatment facility.

We should emphasize that $\mathrm{CT}$ images of these two patients showed typical lesions with ground-glass opacities and consolidations as described in other research (Shi et al., 2020). This indicates the value of chest $\mathrm{CT}$ images in the diagnosis of SARS-CoV-2. In these two cases, absorption of lesions in chest $\mathrm{CT}$ was apparent at the time when RT-CRP of SARS-CoV-2 turned negative for the first time, and the reduction of lesion size was significant (over 50\%). Especially for Patient 1, release of clinical symptoms with apparently improved chest $\mathrm{CT}$ image was not equivalent to no excretion of SARS-CoV-2. According to recent research (Guan et al., 2020; Xia et al., 2020), SARS$\mathrm{CoV}-2$ was not only secreted through the upper repertory tract, but also through the digestive and urinary tracts and the lacrimal passage. A standardized assessment of transmission risk should be based on viral detection of all potential infection routes.

In summary, it may be inappropriate to completely exclude infectious risk in discharged patients based on negative RT-PCR results of upper repertory secretion alone. Clinical evaluation and lung imaging are critical for interpretation of disease progression, while continued viral RT-PCR detection in different specimens would add evidence for evaluating the 
transmission risk of COVID-19 patients. Continuing isolated observation for discharged patients is required and wearing masks with hand hygiene should also be emphasized in these patients for infection control purposes.

\section{Contributors}

Jun-wei SU performed data analysis, wrote and edited the manuscript. Wen-rui WU and Guan-jing LANG performed the data collection. Hong ZHAO participated in the editing of the manuscript. Ji-fang SHENG participated in the study design, writing and editing of the manuscript. All authors have read and approved the final manuscript and, therefore, have full access to all the data in the study and take responsibility for the integrity and security of the data.

\section{Compliance with ethics guidelines}

Jun-wei SU, Wen-rui WU, Guan-jing LANG, Hong ZHAO, and Ji-fang SHENG declare that they have no conflict of interest.

All procedures followed were in accordance with the ethical standards of the responsible committee on human experimentation (institutional and national) and with the Helsinki Declaration of 1975, as revised in 2008 (5). Informed consent was obtained from both patients for being included in the study.

\section{References}

Chan JF, Kok KH, Zhu Z, et al., 2020. Genomic characterization of the 2019 novel human-pathogenic coronavirus isolated from a patient with atypical pneumonia after visiting Wuhan. Emerg Microbes Infect, 9(1):221-236. https://doi.org/10.1080/22221751.2020.1719902

Chang D, Lin MG, Wei L, et al., 2020. Epidemiologic and clinical characteristics of novel coronavirus infections involving 13 patients outside Wuhan, China. JAMA, 323(11): 1092-1093. https://doi.org/10.1001/jama.2020.1623

Fang LQ, Tang F, Fontanet A, et al., 2004. Long-term SARS coronavirus excretion from patient cohort, China. Emerg Infect Dis, 10(10):1841-1843. https://doi.org/10.3201/eid1010.040297

General Office of National Health Commission of the People's Republic of China, 2020. Diagnosis and treatment of pneumonitis caused by new coronavirus (Trial Version 6). http://www.nhc.gov.cn/yzygj/s7653p/202002/8334a8326 dd94d329df351d7da8aefc2.shtml [Accessed on Feb. 20, 2020] (in Chinese).

Guan WJ, Ni ZY, Hu Y, et al., 2020. Clinical characteristics of coronavirus disease 2019 in China. $N$ Engl J Med, in press. https://doi.org/10.1056/NEJMoa2002032

Huang CL, Wang YM, Li XW, et al., 2020. Clinical features of patients infected with 2019 novel coronavirus in Wuhan, China. Lancet, 395(10223):497-506. https://doi.org/10.1016/S0140-6736(20)30183-5

Shi HS, Han XY, Jiang NC, et al., 2020. Radiological findings from 81 patients with COVID-19 pneumonia in Wuhan, China: a descriptive study. Lancet Infect Dis, 20(4):425434. https://doi.org/10.1016/S1473-3099(20)30086-4

World Health Organization, 2020. Coronavirus disease (COVID-19) pandemic. World Health Organization. https:// www.who.int/emergencies/diseases/novel-coronavirus-2019 [Accessed on Apr. 22, 2020].

Xia JH, Tong JP, Liu MY, et al., 2020. Evaluation of coronavirus in tears and conjunctival secretions of patients with SARS-CoV-2 infection. J Med Virol, 92(6):589-594. https://doi.org/10.1002/jmv.25725

Zhu N, Zhang DY, Wang WL, et al., 2020. A novel coronavirus from patients with pneumonia in China, 2019. $N$ Engl J Med, 382:727-733. https://doi.org/10.1056/NEJMoa2001017

\section{中文概要}

\section{题 目: 需谨慎考虑符合出院标准的 2019 冠状病毒病患 者的传染性}

概 要: 目前全球新型冠状病毒流行形势仍严峻且持续恶 化。评估符合出院标准的 2019 冠状病毒病 （COVID-19）患者的传染性对于疾病控制至关 重要。本研究报告两例核酸检测结果与临床表现 及影像学表现不相符的患者, 以此来呼吁临床工 作者谨慎考虑符合出院标准的 COVID-19 患者的 传染性。

关键词：2019冠状病毒病（COVID-19）; 新型冠状病毒 (SARS-CoV-2); 实时聚合酶链式反应(RT-PCR) 